\title{
RACE program provides rapid specialist consults
}

A

British Columbia program that provides family physicians with rapid telephone access to specialists aspires to save money, improve patient care and reduce unnecessary referrals and emergency department visits.

The Rapid Access to Consultative Expertise (RACE) program has facilitated about 7000 calls since its launch in 2010. The majority of those calls, $78 \%$, were answered within 10 minutes, while patients were still in their doctors' offices. A third of patients avoided hospital visits, and $60 \%$ of patients who were candidates for referral avoided visits to specialists.

"For the patient, it's great in terms of managing their medical condition in a more timely way," says Dr. Daniel Dodek, a staff physician at City Square Family Practice in Vancouver, BC. "It's satisfying for me, too, because it gives me the added confidence that I'm doing the right thing."

Dodek uses the RACE program twice a month, on average. He recently consulted with a cardiologist about an 85-year-old female patient with an irregular heartbeat. "I wasn't sure if she needed to be seen urgently in an emergency room by a cardiologist," he recalls. "Based on the phone call, we saved her the aggravation of a hospital visit."

The program, which extends across the Vancouver Coastal Health and Fraser Health regions, allows family doctors to inquire about diagnoses, medication and treatment plans in 17 specialties, including respirology, endocrinology and nephrology. Specialty areas are included based on demand from family physicians, says Margot Wilson, director of the Chronic Disease Management Strategy at Providence Health Care, who helped create RACE.

"We've never been asked to include spinal surgery or urology, but we're always working with new specialty areas to bring on to the RACE line, and have another two that will

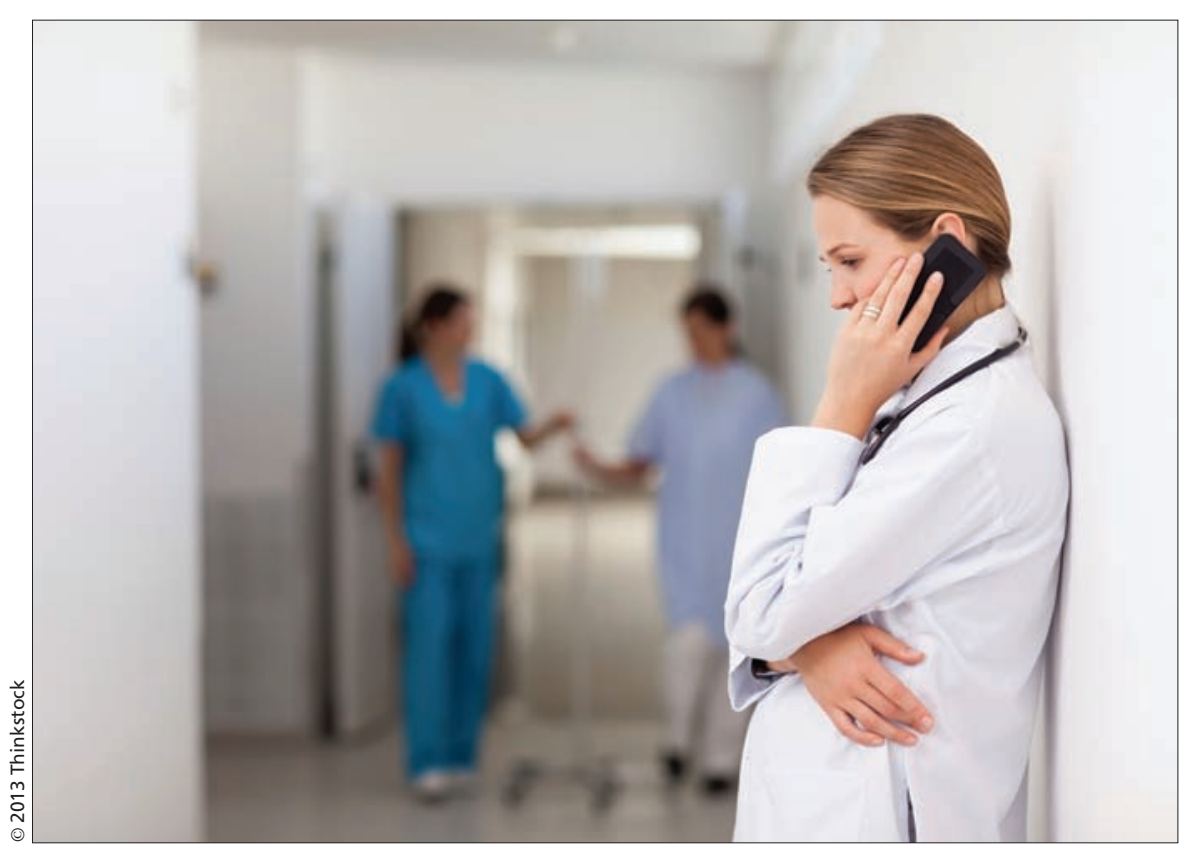

Nearly $\mathbf{8 0} \%$ of calls to specialists in the Rapid Access to Consultative Expertise program are answered within 10 minutes.

likely join in the next few months," says Wilson.

Though a coordinated approach to accessing specialists on a single telephone line is an idea worth adopting, lack of access to particularly hard-toreach specialists is a concern, says Dr. Ernst Schuster, a family physician in Edmonton, Alberta. "But as successful as the RACE program appears in BC, it's interesting that the most difficult specialties to get consultation from are not part of that program: orthopedic surgery, spinal surgery and urology."

Still, the advantages of a RACE-type program impressed Schuster enough to propose the creation of something similar to the Alberta Medical Association, of which he is a board member. He received unanimous support from the board. "The board of directors and staff at AMA need to follow up and see whether we can pull something off," he says.

Health care systems and patients in other provinces would also benefit from programs similar to RACE, says Dr. Lydia Hatcher, a family physician in Hamilton, Ontario.
"The shame is that it's much, much more cost-effective to do a phone consult than have the patient wait for months, with potentially unnecessary tests and hospital visits," she says. "A phone consult is all a patient might need to improve their health."

One area of eastern Ontario, serviced by the Champlain Local Health Integration Network, does have an electronic consultation service, though it isn't for urgent cases. Response times vary from several hours to a week, according to Chantale LeClerc, the network's CEO. But the program, which allows family physicians to contact specialists through a web-based application, has proven useful and convenient, says LeClerc.

"Primary care providers have said it enables them to deliver good plans of care, and in $40 \%$ of cases, specialists were able to avoid face-to-face visits with a patient, freeing them up to care for people who truly need to be seen." - Lauren Kramer, Richmond, BC 